\title{
The role of structural redundancy in the perception of visual targets
}

\author{
R. A. KINCHLA \\ Princeton University, Princeton, New Jersey 08540
}

\begin{abstract}
Most visual images contain "gross shapes" which are highly correlated with less perceptable "details," e.g., the gross outline of a head and details such as eyes, ears, etc. The role of such structural redundancy in perception is considered both from a "syntactical" and "spatialfrequency" view of structure. An experiment is reported and evaluated in terms of a simple mathematical model which allows one to isolate the influence of several factors in the perceptual process, particularly the observer's actual sensitivity to specific details and the influence of the redundant (correlated) information in "higher order forms." The basic idea is that one decides whether a particular (target) detail is present in a briefly presented image by integrating impressions from various parts of the image in a weighted manner, with the weights determined by redundant information in "higher order forms." Furthermore, the results are generally consistent with the notion that an increase in the number of attended letters does not reduce the information extracted from each letter.
\end{abstract}

This paper deals with the relation between "seeing" an object and its parts, or, in more colloquial terms, the relation between "seeing the forest and the trees." The close correlation between an object and its parts is one type of redundancy found in most visual images. Because of such redundancy, it is often difficult to decide whether a particular component was really "seen," in the sense that its contours actually determined one's perception, or whether its presence was unconsciously deduced from perception of a higher order form. For example, if a drawing of a human face is shown to an observer for only a fraction of a second, he will often be convinced he saw a particular detail such as an eye, even if that component had been previously erased from the drawing. In such cases, it seems clear that the more salient or perceptible aspects of the figure, such as the gross contours of the head, defined a higher order form within which an eye was so probable that the viewer simply assumed he had seen it. This paper presents both a theoretical and an experimental approach to the analysis of such perceptual phenomena.

\section{“HIGHER-ORDER FORMS” AND THE CONCEPT OF "STRUCTURE"}

Before going further, it seems useful to briefly consider the notion of structure in visual images, particularly as it relates to the distinction between higher and lower order forms. Psychological ideas about image structure have been strongly influenced by work on computer pattern recognition, especially the syntactical analysis of form summarized by Fu (1974). The basic idea is that forms may be parsed into different levels of structure, much as writing may be parsed into sentences, phrases, and words, etc. Thus a person may be analyzed into parts, such as head, arms, legs, etc., which in turn may be analyzed into even "lower order" parts, such as head into nose, mouth, eyes, etc.

There is another psychologically important basis for distinguishing levels of structure in a visual image which is not adequately captured by the syntactic approach. This will be referred to as the spatial-frequency analysis of form, and can be illustrated most simply as follows. People often distinguish between gross shapes and details in an image. If one were to appropriately "blur" (low-pass, spatial-frequency filter) an image, observers would report that they could still see the gross shapes but could no longer make out the detail. In fact, one could systematically order the components of an image in terms of their recognizability at different levels of "blurring" (cutoff levels of the low-pass filter). The highest order forms would be those which were still identifiable at the highest levels of blurring (lowest filter cutoffs). It is argued here that such a hierarchy of forms has important perceptual properties which the syntactic analysis of forms fails to consider (e.g., see Broadbent, 1977 , for an excellent discussion of this issue).

It should be emphasized that the work presented in this paper was not designed to distinguish between these two views of structure. Nevertheless, it seems useful to point out the possible relevance of spatial frequency analysis of our understanding of image structure. For example, the work of Navon (1975) and Tolhurst (1975) suggests that the low spatial frequency components of an image are 
processed first. This implies an order of processing which is not obvious from a syntactical view of structure.

\section{EXPERIMENTALLY USEFUL STIMULUS PATTERNS}

The problem with using completely "naturalistic" images to study the role of structural redundancy in perception is that the redundancy in such images has usually been learned through long experience and is therefore difficult to manipulate experimentally. Furthermore, much of the redundancy in normal visual images is constrained by natural laws; e.g., there is a biologically appropriate arrangement of the components of a face. A similar problem arises in studying the role of redundancy in reading, since orthographic, syntactic, and semantic rules are so well learned by any literate person so as to seriously constrain their arbitrary revision for experimental purposes. Thus, what is needed is a set of stimuli in which the relationships between higher and lower order forms may be arbitrarily manipulated, and which utilize an observer's highly trained ability to recognize alphabetic forms. A class of stimuli which seem to meet these requirements were initially described' in Kinchla (1974). They are simply "higher order forms" constructed by the spatial arrangement of "lower order forms," specifically, the large letters ( $T$ and $L$ ) defined by the arrangement of smaller letters illustrated in Figure 1. Naturally, if one has sufficient time to view such a display, there is no problem in perceiving either the large or small letters. However, there are tachistoscopic observation intervals of only a few milliseconds at which the small letters are only barely perceptible even though the larger ones remain perfectly legible. At such exposures, it is possible to ask an observer questions about the barely discernible smaller letters, when he knows there is a correlation between the form of each larger letter and the constituent smaller ones; i.e., the gross form of the large letter is made partially redundant with the presence or absence of certain smaller letters. It is argued here that the observer should utilize such redundancy in perceiving the smaller letters in much the same way as he utilizes gross contextual features when process-

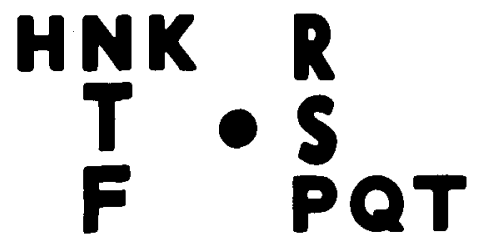

Figure 1. An illustrative stimulus display which can be seen as 2 large letters ( $L$ and $T$ ) or 12 smaller ones. ing the details of any naturally redundant visual image. Here, however, it is possible to manipulate the redundancy at will.

\section{THE BASIC PERCEPTUAL TASK}

For a variety of reasons (discussed later in the paper), it seemed best to employ a simple letter detection procedure to assess the observer's perception of the small letters shown in Figure 1. Specifically, on each of a series of trials, the observer is asked to decide whether or not a particular small letter, the "target letter" $F$, was presented on that trial, answering simply "yes" or "no," without indicating the specific position of the letter. It will be useful to denote a particular (left-right) arrangement of the large $L$ and large $T$ by $L_{j} T_{j}$ or $T_{j} L_{i}$, with the subscript to each letter ( $i$ or $j$ ) equal to one if that large letter contained a small $\mathrm{F}$ and equal to zero otherwise; e.g., the stimulus display illustrated in Figure 1 would be denoted by $T_{1} L_{0}$, since the $T$ is on the left and contains a small $F$. In the particular procedure we shall consider either $L_{i} T_{j}$ or $T_{j} L_{i}$ occurred with equal (.5) probability on each trial. The $T_{0}$ and $\mathrm{L}_{0}$ patterns were composed of the consonants other than $F$, with each consonant being equally likely at any of the six positions within each large letter (the same consonant could occur more than once in each large letter). The $T_{1}$ and $L_{1}$ patterns were composed by randomly replacing one of six consonants in a $\mathrm{T}_{0}$ or $\mathrm{L}_{0}$ pattern with a small $\mathrm{F}$, such that each of the six positions in a $T_{1}$ or $L_{1}$ had an equal (1/6) probability of containing the single target. A partial redundancy (correlation) between the form of each large letter and the presence of the target letter was introduced by manipulating the probabilities that each would contain a target. In the procedure employed in the present experiment, these probabilities, denoted $\mathrm{P}\left(\mathrm{L}_{1}\right)$ and $\mathrm{P}\left(\mathrm{T}_{1}\right)$, were independent so that it was possible for one, neither, or both of the large letters to contain a target $\mathrm{F}$ on each trial; e.g., the probability that both contained a target was simply $P\left(L_{1}\right)$ times $P\left(T_{1}\right)$.

Some things should be emphasized at this point. First, there is no question that a particular target letter might be more perceptible within one large letter than within another, or on one side of the display than on another, simply because of differences in lateral interactions and acuity at various points on the retina. The experiment presented here was designed to control for such differences in order to consider the role of structural redundancy in the perceptual process. Second, alphabetic forms were employed for both "levels" of the display (large and small letters) simply because observers are so highly practiced and proficient at identifying such forms. It is quite possible that this might produce 
unique perceptual problems; e.g., "coding" both large and small forms into the same lexicon might produce conflicts similar to the "Stroop" effect, especially if one of the large letters was identical to a smaller one. It would have been possible to employ nonalphanumeric forms at either or both "levels" of the display; e.g., the small letters could have been arranged to form larger shapes, such as squares or triangles, which could also have been made redundant with the presence of the target. One could even introduce the "higher order" redundancy by arranging the small letters into two identical, but laterally separated, square matrices and coloring each matrix differently, with a target more likely in one color matrix than in another. Each of these variants of the basic task is of interest, even though the experiment we shall consider here employed only stimuli of the sort shown in Figure 1 (letters made up of letters). However the theoretical approach developed in the next section of this paper seems relevant to both the basic task and the variants of it mentioned here.

\section{THEORETICAL APPROACH}

\section{Background}

In a paper originally published as a technical report (Kinchla, Note 1) and subsequently as a journal article (Kinchla, 1969), this author considered the perceptual problem of integrating the information obtained from $n$ observations to decide whether at least one of them was a "signal." Data were presented from an auditory detection task in which the $\mathrm{n}$ observations were made during $\mathrm{n}$, successively defined intervals and the signals were simply pure tones added to a constant background of white noise during randomly selected intervals. Each trial consisted of the $n$ observations $(n=1,3$, or 6), after which the subject made a single response, "yes" or "no," to indicate whether he thought "at least one" of the intervals contained a tone signal (he was not required to specify which intervals contained the signals when he responded "yes"). It was shown that a "d-prime" discriminability measure, $\mathrm{d}_{\mathrm{t}, \mathrm{n}}$, characterized the listener's ability to distinguish trials on which $t$ signals had been presented $(0<t \leqslant n)$ from trials on which no signals had been presented, where

$$
\mathrm{d}_{\mathrm{t}, \mathrm{n}}=\frac{\mathrm{t} \mathrm{d}_{\mathrm{l}, l}}{\mathrm{n}^{1 / 2}}
$$

with $d_{1,1}$ the simple $d^{\prime}$ measure defined by Green and Swets (1966) for a conventional singleobservation detection task. This equation derives from the integration model proposed by this author in which the listener simply sums the impression gained from each observation and responds "yes" only if this sum exceeds some "judgmental criterion"; specifically, if $X_{i}$ is a Gaussian random variable representing the "impression of a signal" evoked by the ith observation, and if $X_{i}$ has the same variance but a larger mean value when interval $i$ contains a signal, then the preceding equation follows, regardless of the specific judgmental criterion. Note that the reduction in one's ability to detect $\mathbf{t}$ signals as $\mathbf{n}$ increases is due to the added variance or "noise" in the "integrated impression" ( $\left(\Sigma \mathrm{X}_{\mathrm{i}}\right)$, not to any change in the relation between $X_{i}$ and the stimulus event in interval $i$ (each observation is made with the equal efficiency). It was proposed then that, while the model had been applied there to a successive observation task: "Similar arguments could be advanced regarding experiments in which an observer was asked to simultaneously monitor two visual stimuli ... or two auditory channels .... Such experiments are of particular interest because it is precisely the question of whether multiple observations can occur simultaneously that is the central issue in the analysis of 'attentional' processes .... The primary point to be made here is that there is an alternative 'nonattentional' interpretation which also accounts for the reduction in accuracy ... [in simultaneous observation tasks]. Specifically, one could argue that the observer makes simultaneous, independent, and equally efficient observations in each channel ... [but his problem] ... is essentially the same as in the preceding multiple-observation analysis [italics added] (Kinchla, 1968, 1969).

A similar proposal was made by Eriksen and Spencer (1969) based on their analyses of a tachistoscopic letter detection task. They concluded that the detectability of a "signal" letter in an array of other letters was the same whether the letters were presented simultaneously or in succession. Shiffrin and Gardner (1972) confirmed Eriksen and Spencer's findings, and Gardner (1973) proposed an "independent channels, unlimited capacity" model which provided a nonattentional interpretation of simultaneous letter processing similar to that suggested by this author for "multiple-channel" tasks. Gardner also presented the results of several excellent experiments which provide strong evidence for the nonattentional interpretation. A more quantitative development and evaluation of a "nonattentional" model applied to a letter-recognition task is presented in an important paper by Shiffrin and Geisler (1973). The letter "recognition" task requires the observer to decide which of two possible "signal" letters was embedded in an array among other "noise" letters, while a "detection" task requires him to decide whether a specific signal letter (or group of letters) was, or was not, presented among an array of "noise" letters. A quantitative evaluation of the simple "integration" or "confusability" model 
(Kinchla, 1969, Note 1) applied to letter detection data is presented in Kinchla (1974), where it is shown that the model provides an excellent interpretation of such data, including some collected by this author and some reported in the previously cited Eriksen and Spencer (1969) paper.

The theoretical significance of these results is obvious. Other, quite influential models had characterized an observer's ability to simultaneously process multiple-letter arrays as an "attentional" problem: He had to share a limited amount of "attention" or "processing capacity" among the letters, thereby reducing the average amount processed from any one letter as the number of letters was increased (e.g., Estes \& Taylor, 1966; Rumelhart, 1970; Sperling, 1963). In contrast, the "nonattentional" (or "unlimited capacity") models represent the observer as integrating information about each letter in the array, which is of the same quality regardless of the number of letters presented (so long as differences due to retinal locus and lateral interactions are adequately controlled; this is usually done by arraying the letters symmetrically around a fixation point and keeping them well separated). The principle difficulty in detecting a signal, then, is simply the increased variance ("noise" or "confusability") contributed to one's "integrated impression" by each additional letter. ${ }^{2}$

While it is intuitively appealing to interpret the information one extracts from each letter in an array as something like a "likelihood ratio" based on a "sensory sample" evoked by that letter, the testable properties of the integration model are much simpler. A basic one is that each letter in the array should have an additive influence on an appropriately scaled measure of the observer's response tendency (specifically, a "Z-transformation"). This aspect of the model is considered in detail in a paper by Kinchla and Collyer (1974), where it is shown that each letter in a four-letter array seemed to have such an "additive" influence on responding in a letter-detection task. However, the additive influence does not imply equal influence, and it was shown that the letters on the left of the array generally had a much stronger influence on responding than those on the right. While one could attribute such differences in influence to differences in the quality of information obtained on the left and right ("acuity" or "noise level" might differ), it is also possible to account for such differences in terms of a weighted integration of the observer's impressions of each letter. This can be stated more formally as follows. In the simple integration model, the probability of responding yes was simply

$$
P(\text { yes })=P\left(\Sigma S_{i}>\beta\right),
$$

where $S_{i}$ was the "impression" obtained of letter $e_{i}$, and B was the "response criterion." The "additive" influence of each letter stems from the summing, while the need to transform the response probabilities to " $Z$ " to demonstrate the additivity stems from the Gaussian variability attributed to each impression. Furthermore, the "effect" of each letter on " $Z$ " is independent of the specific value of the response criterion, $\beta$. The weighted integration model allows the observer to integrate his impressions in a weighted manner so that

$$
P(\text { yes })=P\left(\Sigma \omega_{i} S_{i}>\beta\right),
$$

where each $\omega_{i} \geqslant 0$ and $\Sigma \omega_{i}=1$. The influence of each letter on $\mathrm{Z}$ is still additive, but directly related to $\omega_{\mathrm{i}}$.

Of course, as was pointed out in the Kinchla and Collyer (1974) paper, the weighted integration model is only one way of accounting for differences in each letter's influence. An obvious alternative is to assume an unweighted (or equally weighted) integration, but attribute more variability or "noise" to the impression gained from particular positions in the display. Such an interpretation is one way of characterizing differences in acuity or lateral interference at various points on the retina. Thus, the choice of the weighted integration model would seem to require some way of independently manipulating the weight assigned to one's impression of a particular part of a display and its retinal position; i.e., weighting and position cannot be confounded, or the differential acuity interpretation could equally well apply. The experiment presented in this paper was designed to avoid this problem of confounding and to provide data which to evaluate the following model.

\section{A Weighted Integration Model for Redundancy Effects}

This model represents an observer as integrating his impressions of various parts of the display in a weighted manner, with the weights determined by contextual cues corresponding to "higher order forms." The model will be developed here as if the quality ("noise" level) of the impression gained from a specific large letter is the same whether it is on the left or right side of the display. This is done primarily to simplify the present development, and because it seems adequate for the experimental data considered in this paper; however, the assumption is testable and could be easily modified if the data from $T_{i} L_{j}$ and $L_{j} T_{i}$ displays were significantly different.

The basic assumptions of the model can be summarized verbally as follows:

(1) Let $X_{L}$ denote the observer's "impression" that the large $L$ contained an $F$, and $X_{T}$ his impression that the large $T$ did, where each is an independent, Gaussian random variable whose 
parameters are conditional on $T_{i}$ and $L_{j}$; specifically,

$$
\begin{gathered}
\mathrm{E}\left(\mathrm{X}_{\mathrm{L}} \mid \mathrm{L}_{1}\right)=\mathrm{E}\left(\mathrm{X}_{\mathrm{T}} \mid \mathrm{T}_{1}\right)=1, \\
\mathrm{E}\left(\mathrm{X}_{\mathrm{L}} \mid \mathrm{L}_{0}\right)=\mathrm{E}\left(\mathrm{X}_{\mathrm{T}} \mid \mathrm{T}_{0}\right)=0, \\
\operatorname{Var}\left(\mathrm{X}_{\mathrm{L}}\right)=\alpha_{\mathrm{L}}, \\
\operatorname{Var}\left(\mathrm{X}_{\mathrm{T}}\right)=\alpha_{\Upsilon} .
\end{gathered}
$$

(2) Let the observer report there was at least one small letter $F$ present anywhere in the display if and only if his weighted impression $\mathrm{Y}$ exceeds a response criterion $\beta$, where

$$
Y=\omega X_{L}+(1-\omega) X_{T}
$$

with $0 \leqslant \omega \leqslant 1$.

Note that no assumption is made concerning the degree of interaction between the small letters within each large one, only that $\mathrm{X}_{\mathrm{T}}$ and $\mathrm{X}_{\mathrm{L}}$ are independent and Gaussian. There is considerable evidence that letters located close together are not processed independently (e.g., Wolford, 1975). The two large letters in the present experiment were purposely separated by over $4^{\circ}$ in order to minimize such interactions. Letting the expected value of each impression equal 1 if the large letter contained an $F$ and equal 0 otherwise is primarily an arbitrary choice of origin and unit. Differences in the quality of the impressions from the $\mathrm{L}$ and $\mathrm{T}$ are characterized solely by their respective "noise" levels, $\alpha_{\mathrm{L}}$ and $\alpha_{\mathrm{T}}$ (e.g., the lateral interactions between the components within each letter might differ systematically so that $\alpha_{\mathrm{L}} \neq \alpha_{\mathrm{T}}$, Equations 3 and 4). And, finally, the integration of information from each large letter is represented as a weighted average with the weighting parameter $\omega$ denoting the relative weight assigned to $X_{L}$. Presumably the $\omega$ values are assigned as the observer learns the conditional probabilities of targets within the two large letters, i.e., "learns the structural redundancy."

\section{Properties of the Model}

The testable properties of the model can be specified most easily in terms of the "influence" of the large $L$ or large $T$ on an observer's response tendencies, denoted, respectively, $\mathrm{I}_{\mathrm{L}}$ and $\mathrm{I}_{\mathrm{T}}$. Basically these are simply the additive effects of presenting a target (F) in each large letter (given the appropriate transformation of the object's response tendencies). More specifically, let

$$
I_{L}=\frac{E\left(Y \mid L_{1} T_{1}\right)-E\left(Y \mid L_{0} T_{1}\right)}{[\operatorname{Var}(Y)]^{1 / 2}}
$$

$$
\begin{aligned}
& =\frac{E\left(Y \mid L_{1} T_{0}\right)-E\left(Y \mid L_{0} T_{0}\right)}{[\operatorname{Var}(Y)]^{1 / 2}} \\
& =\frac{\omega}{\left[\omega^{2} \alpha_{L}+(1-\omega)^{2} \alpha_{T}\right]^{1 / 2}},
\end{aligned}
$$

and

$$
\begin{aligned}
\mathrm{I}_{\mathrm{T}} & =\frac{\mathrm{E}\left(\mathrm{Y} \mid \mathrm{L}_{1} \mathrm{~T}_{1}\right)-\mathrm{E}\left(\mathrm{Y} \mid \mathrm{L}_{1} \mathrm{~T}_{0}\right)}{[\operatorname{Var}(\mathrm{Y})]^{1 / 2}} \\
& =\frac{\mathrm{E}\left(\mathrm{Y} \mid \mathrm{L}_{0} \mathrm{~T}_{1}\right)-\mathrm{E}\left(\mathrm{Y} \mid \mathrm{L}_{0} \mathrm{~T}_{0}\right)}{[\operatorname{Var}(\mathrm{Y})]^{1 / 2}} \\
& =\frac{1-\omega}{\left[\omega^{2} \alpha_{L}+(1-\omega)^{2} \alpha_{\mathrm{T}}\right]^{1 / 2}} .
\end{aligned}
$$

Note that $I_{L}$ is simply the difference between the expected value of $X_{L}$ given an $F$ in the $L$, and its expected value given no $F$ in the $L$, expressed in units of the standard deviation of $Y$. Note also that neither $I_{L}$ nor $I_{T}$ depends on the value of $\beta$, the "response criterion," since $\beta$ is not a parameter in Equations 6 and 7 . The relation of these "influence measures" ( $I_{L}$ and $I_{T}$ ) to an observer's theoretical response probabilities $\left(\mathrm{P}_{\mathrm{ij}}\right)$ can be illustrated most simply in terms, of the graph shown in Figure 2. Note that the response probabilities have been scaled such that

$$
P_{i j}=1-\phi\left(Z_{i j}\right),
$$

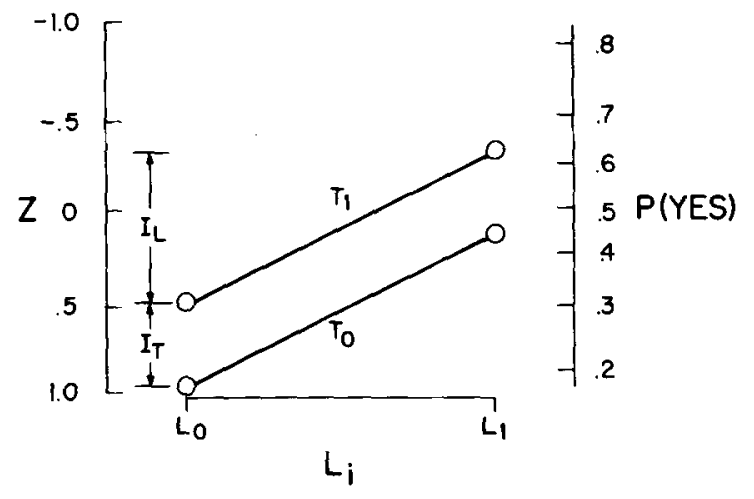

Figure 2. An illustrative graph of the four response probabilities, $P_{i j}$, on a cumulative Gaussian scale $\left(Z_{\mathrm{ij}}\right)$, indicating the "additive" influence of targets in each large letter. This $I_{T}$ is the increase in response tendency $(Z)$ produced by including a target in the large $T$, whereas $I_{L}$ is the increase produced by including a target in the large $L$. 
where $\phi$ is the cumulative standard normal function; i.e., $Z_{i j}$ is that value of a standard normal (' $Z$ ') variable whose cumulative function equals one minus $P_{i j}$. Thus $Z_{i j}$ is simply a transformation of $P_{i j}$. The virtue of this transformation is that, according to the model, the presence or absence of an F in each large letter should have an additive effect on $\mathrm{Z}_{\mathrm{ij}}$, whereas the corresponding effects on $\mathrm{P}_{\mathrm{ij}}$ are not additive. As seen in Figure 2, $I_{L}$ is simply the change in $Z_{i j}$ produced by presenting a target in the large $L$ $\left(Z_{0 j}-Z_{1 j}\right.$ for $\left.j=0,1\right)$, while $I_{T}$ is the increment produced by presenting a target in the large $T$ $\left(Z_{i 0}-Z_{i 1}\right.$ for $\left.i=0,1\right)$. These effects are "additive" in the sense that $Z_{00}-Z_{11}$ is simply the sum of $I_{L}$ and $I_{T}$. These properties follow directly from the definitions of $I_{L}$ and $I_{T}$, Equations 6 and 7 , and the basic assumptions of the model, Equations 1-5; specifically,

$$
\begin{aligned}
P_{i j} & =P\left(Y \geqslant \beta \mid L_{i} T_{j}\right) \\
& =1-\phi\left(Z_{i j}\right),
\end{aligned}
$$

where

$$
Z_{i j}=\frac{\beta-E\left(Y \mid L_{i} T_{j}\right)}{[\operatorname{Var}(Y)]^{1 / 2}} .
$$

Thus, it follows that

$$
\begin{aligned}
\mathrm{I}_{\mathrm{L}} & =\mathrm{Z}_{01}-\mathrm{Z}_{11} \\
& =\mathrm{Z}_{00}-\mathrm{Z}_{10}, \\
\mathrm{I}_{\mathrm{T}} & =\mathrm{Z}_{10}-\mathrm{Z}_{11} \\
& =\mathrm{Z}_{00}-\mathrm{Z}_{01},
\end{aligned}
$$

and

$$
\mathrm{I}_{\mathrm{L}}+\mathrm{I}_{\mathrm{T}}=\mathrm{Z}_{00}-\mathrm{Z}_{11}
$$

The relationship between $I_{L}$ and $I_{T}$ is of particular interest and can be obtained directly by adding Equations 6 and 7 and rearranging as follows:

$$
I_{L}=\frac{1}{\left[\omega^{2} \alpha_{L}-(1-\omega)^{2} \alpha_{T}\right]^{1 / 2}}-I_{T}
$$

A graphic illustration of this relationship is presented in Figure 3 for the special case in which $\alpha_{\mathrm{L}}$ equals $\alpha_{\mathrm{T}}$ equals .6. Note that the influence of the large $L$ is maximal when $\omega$ equals one, while the influence of the large $T$ is maximal when $\omega$ equals zero, with intermediate values of $\omega$ producing intermediate combinations of $I_{L}$ and $I_{T}$. Of course, it would be possible for $\alpha_{\mathrm{L}}$ and $\alpha_{\mathrm{T}}$ to have different values, in contrast to the case illustrated in Figure 3 (where $\alpha_{\mathrm{L}}=\alpha_{\mathrm{T}}=.6$ ). However, the relation between $I_{L}$ and $I_{T}$ would have the same general form: a negatively accelerating function from a point on the ordinate defined when $\omega$ equals one, to one on the abscissa defined when $\omega$ equals zero. It will be useful to denote these two points as $\mathrm{D}_{\mathrm{L}}$ and $\mathrm{D}_{\mathrm{T}}$, respectively, where, by Equations 6, 7, and 12:

$$
\mathrm{D}_{\mathrm{L}}=\frac{1}{\alpha_{\mathrm{L}} 1 / 2}
$$

and

$$
\mathrm{D}_{\mathrm{T}}=\frac{1}{\alpha_{\mathrm{T}} 1 / 2}
$$

Thus $\mathrm{D}_{\mathrm{L}}$ characterizes the observer's ability to detect a target in the large $L$ when he bases his response solely on $\mathrm{X}_{\mathrm{L}}(\omega=1)$, and $\mathrm{D}_{\mathrm{T}}$ is a similar measure of his ability to detect a target in the large $\mathrm{T}$ using only $X_{T}(\omega=0)$. For this reason, $D_{L}$ and $D_{T}$ will be termed the "discriminability" of a target in the large $L$ or $T$, respectively. Note that $D_{L}$ and $D_{T}$ depend only on $\alpha_{\mathrm{L}}, \alpha_{\mathrm{T}}$, and not on the response criterion $\beta$, or the weighting parameter $\omega$.

Finally, it is useful to note that Equations 6 and 7 imply that

$$
\frac{I_{L}}{I_{T}}=\frac{\omega}{1-\omega},
$$

or, rearranging terms,

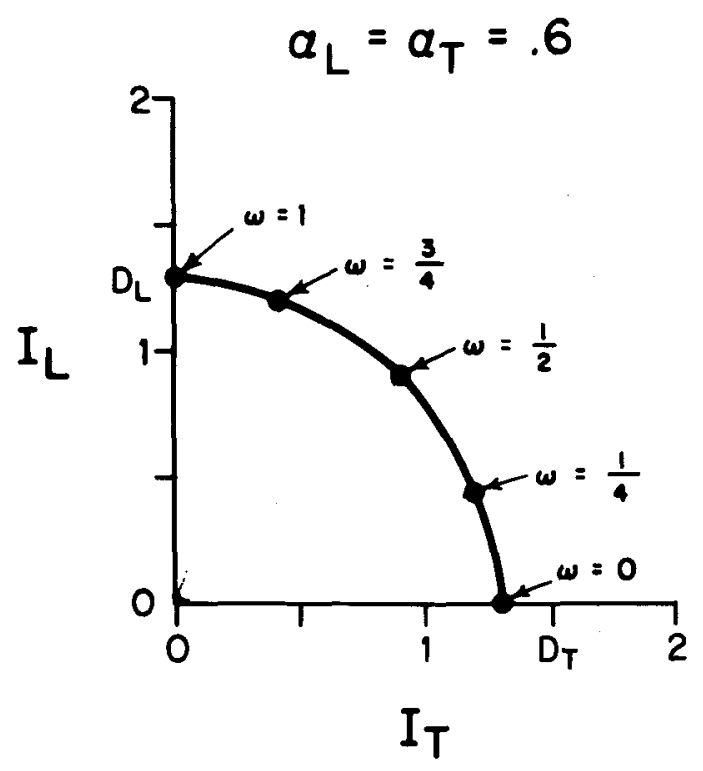

Figure 3. The relation between the influence measures $I_{L}$ and $I_{T}$ (Equation 12) for the special case in which $\alpha_{L}$ equals $\alpha_{T}$ equals .6. 


$$
\omega=\frac{\mathrm{I}_{\mathrm{L}}}{\mathrm{I}_{\mathrm{L}}+\mathrm{I}_{\mathrm{T}}} .
$$

This relation provides a simple way of estimating $\omega$ from estimates of $I_{L}$ and $I_{T}$.

\section{Should $D_{\mathbf{L}}$ and $D_{\mathrm{T}}$ Depend on $\omega$ ?}

An important feature of the model is that $\alpha_{\mathrm{L}}$ and $\alpha_{\mathrm{T}}$ ("noise" in the processing of the large $\mathrm{L}$ and $\mathrm{T}$, respectively) are independent of $\omega$; i.e., "discriminability" of a target in each letter $\left(\mathrm{D}_{\mathrm{L}}\right.$ and $\left.\mathrm{D}_{\mathrm{T}}\right)$ should be invariant under changes in $\omega$. However, one could question this aspect of the model as follows. Suppose the observer were told to base his response solely on the presence or absence of a target in the large $\mathrm{L}$. He should then concentrate all his "processing capacity" on the details of the large $L$, and base his response solely on $\mathrm{X}_{\mathrm{L}}(\omega=1)$. Similarly, if told to consider only the large $T$, he might "concentrate" on the details of the large $T(\omega=0)$. However, suppose his processing capacity were limited, so that he was unable to process the details of both large letters on the same trial as effectively as he could only one. If this were the case, one would expect at least one of the "noise levels," $\alpha_{\mathrm{L}}$ or $\alpha_{\mathrm{T}}$, to be higher when $\omega$ was between 0 and 1 than when it equaled 0 or 1 ; i.e., discriminability of the target in at least one of the large letters $\left(D_{L}\right.$ or $\left.D_{T}\right)$ should be lower when the observer was told to report a target in either large letter $(0<\omega<1)$, rather than to report a target only if it were in a specific letter $(\omega=0,1)$. The major point to be made here can be stated as follows: Assuming the same "weighted integration" process, a "capacity limitation" of this sort would result in performances (combinations of $I_{L}$ and $I_{T}$ ) somewhere under the function relating $I_{L}$ and $I_{T}$ defined by Equation 12 (and illustrated in Figure 3), so long as $\alpha_{L}$ and $\alpha_{T}$ were estimated from conditions in which $\omega$ equaled 1 and 0 , respectively.

\section{EXPERIMENT}

This experiment assessed the influence of three factors on performance of the detection task described earlier in this paper. These three independent variables will be termed the "stimulus schedule," the "criterial instruction," and the "task definition." The stimulus schedule was either: Schedule A, in which targets were twice as likely in the large $L$ as in the large $T, P\left(L_{1}\right)$ equal .5 and $P\left(T_{1}\right)$ equal .25; or, Schedule $B$, in which just the opposite was true, $P\left(L_{1}\right)$ equal .25 , and $P\left(T_{1}\right)$ equal .5 (note that the overall proportion of trials on which ' at least one target was presented was the same under either schedule, .625). The criterial instruction consisted of telling the observer either to be "liberal" in reporting targets ("say yes if you're even slightly sure you saw one") or to be "conservative" ("don't say yes unless you're quite sure you saw one"). And finally, the task definition took one of three forms: telling the observer to report an F only if it occurred in the large $T$, "T only"; only if it occurred in the large L, "L only"; or if it occurred in either large letter "either letter." Thus, there were a total of 12 experimental conditions defined by the factorial combinations of these three variables ( 2 by 2 by 3 ). It was anticipated that each factor would have a substantial influence on an observer's performance, and the central question was whether the "weighted integration" model provides a useful framework for interpreting these effects.

\section{Procedure}

Three subjects were tested extensively on the previously described letter detection task, i.e., they tried to detect the presence of a small letter " $F$ " among the 12 small letters defining a large " $T$ " and " $L$." Each test trial consisted of a brief, 10-msec presentation of a display, a 2 -sec response period in which they made either a "yes" or a "no" response (they were not asked to indicate the position of the $F$ when they responded yes); and finally a 1-sec "feedback" period during which the same display was visible to allow them to check the accuracy of their response. There was a $2-\mathrm{sec}$ intertrial interval between each trial, when only a fixation point was visible. The displays were all of the same general form as that shown in Figure 1, except that, as indicated earlier, the relative, left-right position of the large $L$ and $T$ was varied randomly from trial to trial along with the constituent small letters. The subjects viewed the displays binocularly, with each large letter centered about $4^{\circ}$ from the fixation point. The stimuli were presented on a CRT display (Tektronix Model 610) under the control of a laboratory computer (DEC-PDP 12), using its standard letter display format. The computer also recorded the observers' "pushbutton" responses.

Each of the three observers performed a total of 600 trials under each of the 12 experimental conditions, yielding a total of 7,200 trials of data from each observer. Each subject performed 100 unrecorded "practice" trials under each of the 12 conditions prior to actual data collection. Data was then collected in a series of 24 daily 300 -trial experimental sessions. A single experimental condition was in effect throughout each session, with an appropriately counterbalanced ordering of conditions.

\section{Results}

Each of the three major experimental variables had a statistically significant $\left(\chi^{2}, \mathrm{p}<.001\right)$, and substantial, influence on each observer's performance. The pattern of these effects was the same for each observer. It can be seen in Figure 4, which presents the proportion of $R_{1}$ ("yes") responses to each stimulus pattern under each condition, averaged over the three observers. (Since there were no significant differences in performance due to the left-right arrangement of $L_{i}$ and $T_{j}$, data from $L_{i} T_{j}$ and $T_{j} L_{i}$ trials were combined for each combination of $i$ and j.) Note that the subjects were forced to respond $R_{1}$ or $R_{0}$, on every trial so that the proportions of $R_{1}$ responses to each stimulus pattern completely summarize their performances. An $\mathbf{R}_{\mathbf{t}}$ response is a "correct" response whenever one of the large letters 
they were asked to consider contained a target, e.g., $\mathrm{T}$ or $\mathrm{L}$ in the "either" condition, but only $\mathrm{T}$ in the "T-only" condition.

The major effects of the experimental manipulations on each observer's performance are readily apparent in Figure 4, and can be stated verbally as follows:

(1) Under the "L-only" and "T-only" task definitions, only the presence or absence of a target in the designated large letter had a consistent influence on the observers' tendencies to say "yes" $\left(R_{1}\right)$.

(2) Under the "either" task definition, the observers' response tendencies were clearly influenced by the presence or absence of targets in both large letters. However, the relative influence of targets in each letter seems to depend on the schedule (A or B): specifically, the presence or absence of a target in the large letter most likely to contain a target ( $\mathrm{L}$ under Schedule A and T under Schedule B) had the larger effect on the proportion of $R_{1}$ responses.

(3) While the "liberal" criterial instructions clearly produce more $R_{1}$ responses than the "conservative" instructions, the pattern of results was otherwise exactly the same.

A more detailed interpretation of these effects is possible in terms of the weighted-integration model.

\section{Theoretical Analysis}

Estimates of the two "influence" measures, $I_{L}$ and $I_{T}$, were obtained from the performance of each observer in each of the 12 experimental conditions, with each performance defined by the observed proportions of $R_{1}$ responses to each of the four stimulus patterns. These were used as estimates $\left(\hat{p}_{i j}\right)$ of the $p_{i j}$ values in Equation 8, which then implied estimates $\left(Z_{i j}\right)$ of the $Z_{i j}$ values in the same equation. Estimates of $I_{L}$ and $I_{T}$ were then defined as

$$
\hat{\mathbf{I}}_{\mathrm{L}}=\sum_{\mathrm{j}}\left(\hat{\mathrm{Z}}_{0 \mathrm{j}}-\hat{\mathrm{Z}}_{\mathrm{lj}}\right) / 2
$$

and
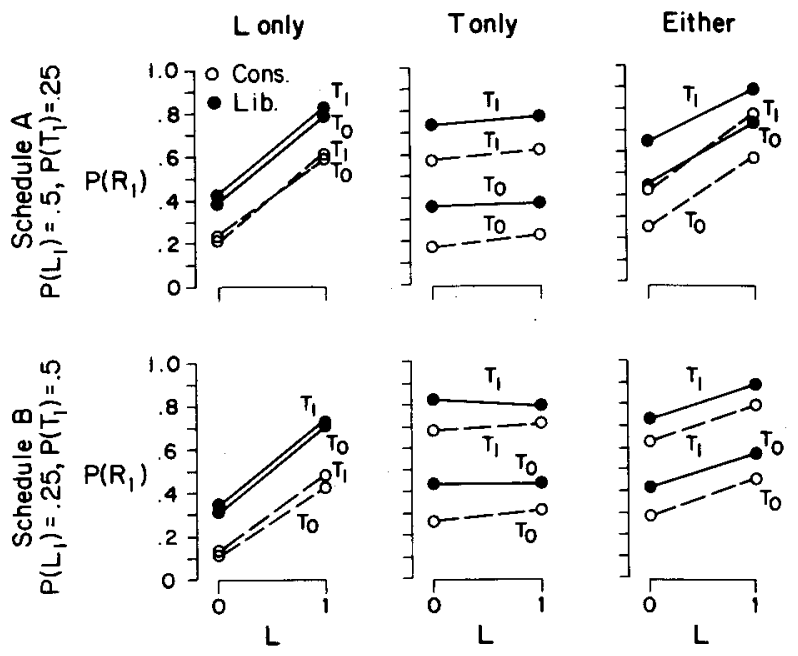

Figure 4. The average proportion of $R_{1}$ responses by the three observers to each of the four stimulus patterns $\left(L_{i} T_{j}\right)$ in each condition.

$$
\hat{\mathbf{I}}_{\mathbf{T}}=\sum_{\mathrm{i}}\left(\hat{\mathrm{Z}}_{\mathrm{i} 0}-\hat{\mathrm{Z}}_{\mathrm{i} 1}\right) / 2
$$

This algebraic procedure corresponds to plotting the four $\hat{p}_{\mathrm{ij}}$ values as shown in Figure 2 and selecting the best fitting (in a least squares sense) pair of parallel lines. (More elaborate estimation procedures might have been employed, but this procedure seemed adequate for the present analysis.) The obtained values of $\hat{I}_{\mathrm{L}}$ and $\hat{\mathrm{I}}_{\mathrm{T}}$ are presented numerically in Table 1 and graphically in Figure 5, which presents a plot of $\hat{\mathrm{I}}_{\mathrm{L}}$ against $\hat{\mathrm{I}}_{\mathrm{T}}$ for the 12 experimental conditions, for each observer. The solid curves on each graph are the theoretical function relating $\mathrm{I}_{\mathrm{L}}$ and $\mathrm{I}_{\mathrm{T}}$ defined by Equation 12 with $\alpha_{\mathrm{L}}$ equal to $\alpha_{\mathrm{T}}$ equal to $\hat{\alpha}$. The value of $\hat{\alpha}$ was chosen for each observer to minimize the average square distance between the 12 data points and their nearest points on the function.

First of all, note that the simple assumption that $\alpha_{\mathrm{L}}$ equals $\alpha_{\mathrm{T}}$ seems reasonable for all three observers.

Table 1

\begin{tabular}{|c|c|c|c|c|c|c|c|c|c|c|c|c|}
\hline & \multicolumn{4}{|c|}{$\mathrm{F}$ in L only } & \multicolumn{4}{|c|}{$\mathrm{F}$ in $\mathrm{T}$ only } & \multicolumn{4}{|c|}{$F$ in either } \\
\hline & \multicolumn{2}{|c|}{ Cons } & \multicolumn{2}{|c|}{ Lib } & \multicolumn{2}{|c|}{ Cons } & \multicolumn{2}{|c|}{ Lib } & \multicolumn{2}{|c|}{ Cons } & \multicolumn{2}{|c|}{ Lib } \\
\hline & $\mathbf{A}$ & B & A & B & A & B & $\mathbf{A}$ & B & A & B & $\mathrm{A}$ & B \\
\hline $\operatorname{Obs}{ } \mathbf{I}_{\mathbf{T}}^{\mathbf{I}_{\mathbf{L}}}$ & $\begin{array}{l}.98 \\
.15\end{array}$ & $\begin{array}{l}.92 \\
.10\end{array}$ & $\begin{array}{l}.81 \\
.11\end{array}$ & $\begin{array}{l}.93 \\
.14\end{array}$ & $\begin{array}{l}.08 \\
.83\end{array}$ & $\begin{array}{l}.09 \\
.90\end{array}$ & $\begin{array}{l}.04 \\
.90\end{array}$ & $\begin{array}{r}.05 \\
1.01\end{array}$ & $\begin{array}{l}.82 \\
.50\end{array}$ & $\begin{array}{l}.51 \\
.78\end{array}$ & $\begin{array}{l}.74 \\
.51\end{array}$ & $\begin{array}{l}.56 \\
.75\end{array}$ \\
\hline $\operatorname{Obs} 2{\underset{\mathbf{I}}{\mathbf{T}}}_{\mathbf{I}}^{\mathbf{I}_{\mathbf{L}}}$ & $\begin{array}{r}1.15 \\
.04\end{array}$ & $\begin{array}{r}1.11 \\
.12\end{array}$ & $\begin{array}{r}1.23 \\
.05\end{array}$ & $\begin{array}{r}1.10 \\
.03\end{array}$ & $\begin{array}{r}.20 \\
1.27\end{array}$ & $\begin{array}{r}.08 \\
1.30\end{array}$ & $\begin{array}{r}.02 \\
1.20\end{array}$ & $\begin{array}{r}.03 \\
1.29\end{array}$ & $\begin{array}{r}1.00 \\
.61\end{array}$ & $\begin{array}{r}.46 \\
1.11\end{array}$ & $\begin{array}{l}.92 \\
.63\end{array}$ & $\begin{array}{r}.68 \\
1.18\end{array}$ \\
\hline Obs $3{ }_{I_{T}}^{I_{L}}$ & $\begin{array}{r}1.05 \\
.04 \\
\end{array}$ & $\begin{array}{r}1.11 \\
.14\end{array}$ & $\begin{array}{r}1.22 \\
.14\end{array}$ & $\begin{array}{r}1.18 \\
.03\end{array}$ & $\begin{array}{r}.06 \\
1.17 \\
\end{array}$ & $\begin{array}{r}.10 \\
1.28 \\
\end{array}$ & $\begin{array}{r}.09 \\
1.19\end{array}$ & $\begin{array}{r}.04 \\
1.23\end{array}$ & $\begin{array}{l}.85 \\
.47\end{array}$ & $\begin{array}{r}.59 \\
1.06\end{array}$ & $\begin{array}{l}.97 \\
.62\end{array}$ & $\begin{array}{r}.48 \\
1.09\end{array}$ \\
\hline
\end{tabular}

"Influence" of Large $L\left(\hat{\mathbf{A}}_{L}\right)$ and Large $T\left(\hat{\mathfrak{A}}_{T}\right)$ Under Each of the 12 Experimental Conditions for Each Observer 
OBs. 1

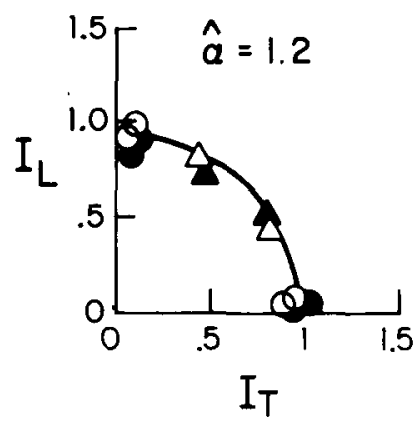

2

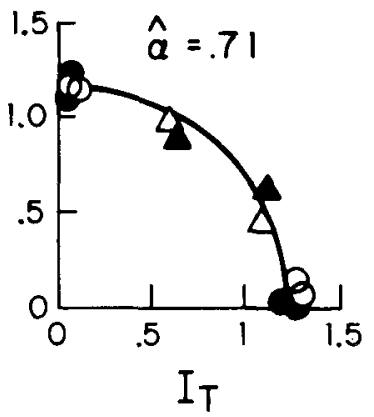

3

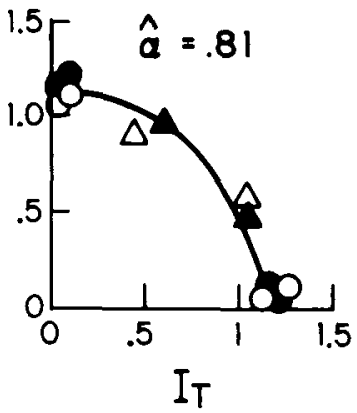

Figure 5. The relation between $\hat{\mathbf{I}}_{\mathrm{L}}$ and $\hat{\mathbf{I}}_{\mathrm{T}}$ for each observer in each condition (values of $\hat{\mathbf{I}}_{\mathbf{L}}$ and $\hat{\mathbf{l}}_{T}$ in Table 1). The open points denote "liberal" criterial conditions and the solid, "conservative." The circles denote " $L$ only" (larger $\hat{\mathbf{I}}_{L}$ values) or "T only" (larger $\hat{\mathbf{I}}_{\mathbf{I}}$ values). The triangles denote the "both" conditions, with the two larger $\hat{i}_{L}$ values corresponding to Schedule $A$ in which targets were more likely to occur in the large $L$. The solid lines are a "least squares" fit of Equation 12 assuming $\alpha_{L}$ equal $\alpha_{T}$ equal $\alpha$.

The cluster of four circular points near the ordinate on each graph in Figure 5 correspond to the "L-only" task conditions, while the four circular points near the abscissa correspond to the "T-only" conditions. Not only are these eight points consistent with the assumption that $\alpha_{\mathrm{L}}$ equals $\alpha_{\mathrm{T}}$ equals $\alpha$, but so are the four triangular points which are plotted in the center of each graph. In other words, there is no evidence that the quality of processing within each large letter (the "noise levels" $\alpha_{\mathrm{L}}$ and $\alpha_{\mathrm{T}}$ ) was influenced by any of the experimental manipulations. Thus the potential capacity problem discussed earlier is not supported by the data; specifically, there is no indication that an observer has more difficulty in processing both large letters of the same trial than he has processing either letter alone. As indicated earlier, a capacity problem of this sort should produce pairs of $\hat{\mathrm{I}}_{\mathrm{L}}$ and $\hat{\mathrm{I}}_{\mathrm{T}}$ values in the "either-letter" condition (triangular points in Figure 5) which fall under the function (Equation 12) suggested by the "Tonly" or "L-only" data (circular points in Figure 5). As can be seen in Figure 5, performance in all 12 conditions seems consistent with a single estimate of $\alpha$ for each observer, including those from the "liberal" (solid points) and "conservative" (open points) criterial instruction conditions. ${ }^{3}$

In terms of the model, the effects of each experimental manipulation can be attributed to changes in the weighting parameter, $\omega$, and the response criterion, $\beta$. Figure 6 presents estimates of the weighting parameter for each observer in each of the 12 experimental conditions. Each estimate is based on Equation 15 and the observed values of $\hat{\mathrm{I}}_{\mathrm{L}}$ and $\hat{\mathrm{I}}_{\mathrm{T}}$ for that observer in that condition. When the observers were instructed to report a small $F$ "only if it occurred in the large T," they appeared to base their responses almost solely on $\mathrm{X}_{\mathrm{T}}$, since estimates of $\omega$ were all close to zero (although consistently greater
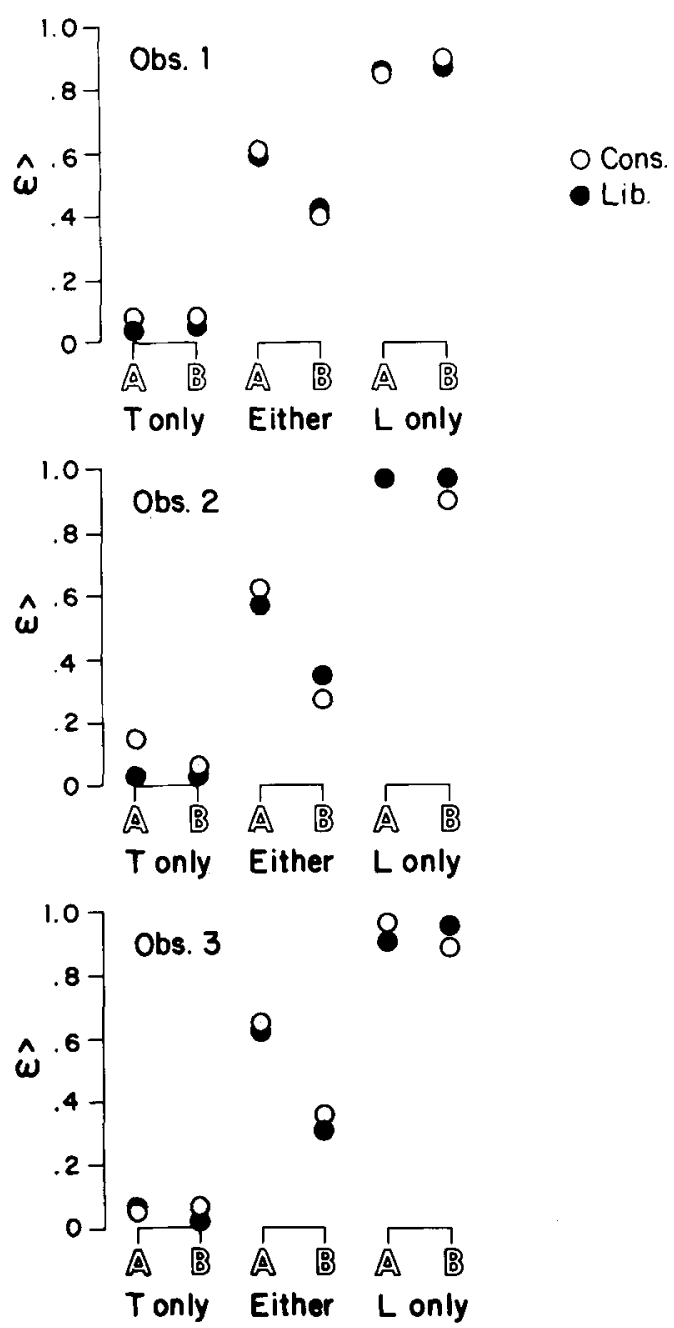

Figure 6. Estimates of the weighting parameter, $\omega$, for each subject in each condition based on Equation 12 and the values of $\hat{\mathbf{L}}_{\mathbf{L}}$ and $\hat{\mathbf{I}}_{\mathbf{T}}$ in Table $\mathbf{1}$. 
than zero, as if $\mathrm{X}_{\mathrm{T}}$ had a small influence in spite of the instructions). Conversely, when told to base their responses on the presence of a target in the large "L only," they all adopted values of $\omega$ close to one (although slightly less than one, as if $X_{L}$ still had some small influence). When told to report a small F occurring in "either large letter," the observers adopted intermediate values of $\omega$, whose relative magnitude reflected the relative probability of a small $F$ being contained in each large letter; $\hat{\omega}$ was close to .6 , given Schedule $\mathrm{A}$, in which an $\mathrm{F}$ was twice as likely in the large $\mathrm{L}$ as in the large $\mathrm{T}$, and close to .3 or .4 , given Schedule $B$, in which an $F$ was twice as likely in the large $T$. Finally, the instructions to be "conservative" (open points) or "liberal" (solid points) had no apparent systematic influence on $\omega$.

Figure 7 present estimates of the response criterion, $\hat{\beta}$, for each observer in each condition based on the $\hat{Z}_{i j}$ and $\hat{\omega}$ values (Figure 6 ) for that condition, and relation defined by Equation 9. (Again, while more elaborate estimation procedures might be employed, this procedure adequately characterizes the general theoretical implications of the data regarding $\hat{\omega}$ and $\hat{\beta}$.) The major implication of Figure 7 is quite clear, the instructions to be "liberal" (solid points) or "conservative" (open points) had a consistent and substantial influence on $\hat{\beta}$, even though it had no consistent effect on $\hat{\omega}$ (Figure 6). Each observer appeared to adopt a lower response criterion $(\beta)$ for reporting a target when told to be "liberal" rather than "conservative." A lower criterion was also adopted in the "T-only" and "L-only" conditions, given schedules in which the specified letter had its higher probability of containing a target: i.e., Schedule A for "T only" and B for "L only."

\section{DISCUSSION}

The proposed model seems to provide a simple, quantitative interpretation of how one type of image redundancy influences our perceptual process. The present development is not intended as a strong test of this model vs. an alternative, but rather a demonstration of one experimental and theoretical approach to the analysis of structural redundancy effects in perception. The relevance of this approach can most easily be considered in reference to other lines of related research.

\section{Related Research}

One important line of related research deals with the role of orthographic redundancy in the recognition of tachistoscopically presented letter strings: the so-called "word-superiority effect," whereby a letter seems more perceptible when part of a word rather than an unrelated string of letters (e.g., Wheeler, 1970). This effect persists even when one attempts to control for it by restricting an observer to a "forced-choice" decision between two subsequently presented letters, both equally consistent with the "word" context. One explanation for this is that the observer automatically proceeds to "code" or "identify" the test letter based on his initial processing of its detail, and loses (forgets) this detail before the forced-choice alternatives are specified. Since a "word" context would facilitate the initial letter identification, the identification would more likely match a forced-choice alternative in a word context. Estes (1975) presents rather convincing evidence for this view by showing that the word-superiority effect disappears if the forced-choice alternatives

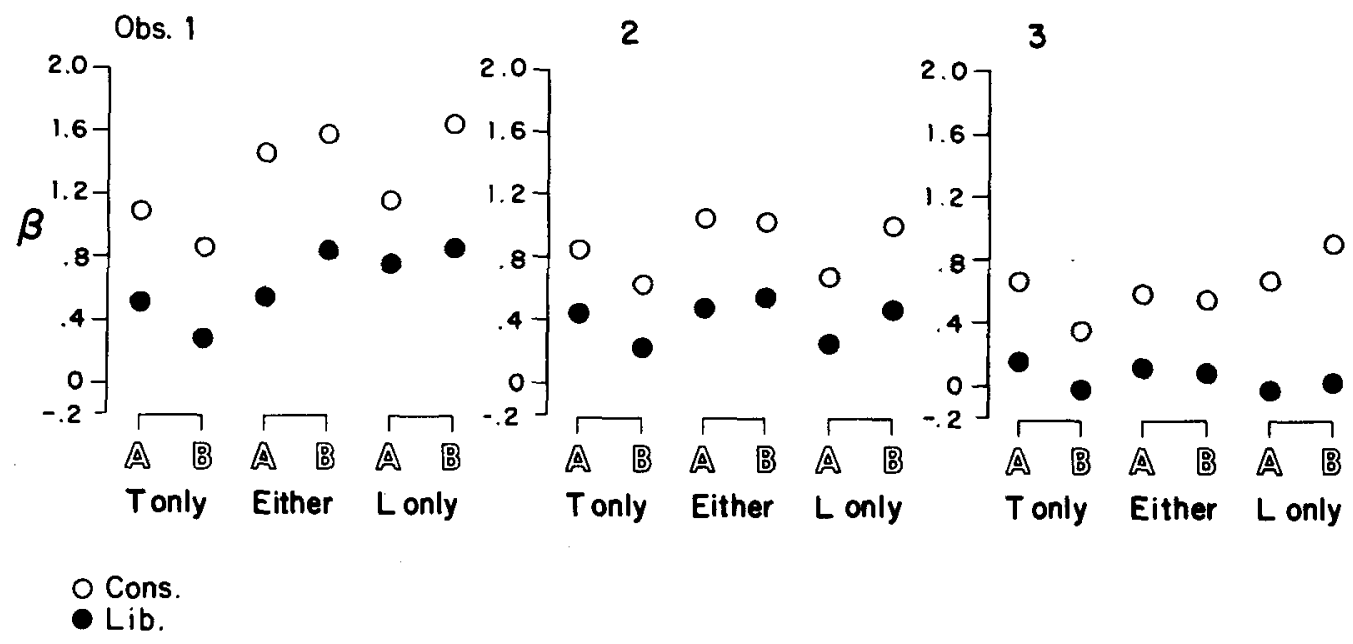

Figure 7. Estimates of response criterion, $\dot{\beta}$, for each subject in each condition, based on Equation 6 and the estimates of $Z_{\mathrm{ij}}$ (see Equation 8) and $\omega$ (Figure 6). 
are defined prior to the test stimuli (although Johnston and McClelland, 1974, found evidence that "attending" to single letters rather than the whole word may alter the perceptual process). The idea that perception proceeds rather automatically to an identification, with rapid loss of the initially processed detail ("features"), suggests that contextual redundancy effects would be most effective during or immediately following a display. If so, the redundant information in the large letters must be rapidly processed and utilized in identifying the smaller letters. Schneider and Shiffrin (1977) and Shiffrin and Schneider (1977) have proposed that tasks involving repeated identification of the same target letter produce an "automatic attention response" which demands very little "processing capacity." However, they point out that automatic attention is unlikely to develop in threshold tasks because the inevitable inconsistency in mapping would prevent such learning. In any case, while the present study involved repeated identification of the same target, it is clear that its detection involved processing both the form of the large letter and the target itself. It is also tempting to characterize the model presented here as "nonattentional," in that the quality (noise levels) of the "impressions," $X_{L}$ and $X_{T}$, do not seem to be altered by the experimental manipulations, only the "integration" of these impressions. However, a selective weighting of information is consistent with a broader view of attention, since the influence of those regions of the display which are given low weights is diminished just as much as if the observer were less sensitive to those regions. Furthermore, having made his decision, the observer has essentially "coded" the information represented in $X_{L}$ and $\mathrm{X}_{\mathrm{T}}$ into a binary code ("yes or no"), and only that code may be accessible later in time. If so, the selective weighting would determine how much information in $\mathrm{X}_{\mathrm{L}}$ or $\mathrm{X}_{\mathrm{T}}$ was retained by the observer.

Palmer (1975) demonstrated the effect of context on the visual identification of objects by clearly presenting a contextual scene prior to a barely perceptible target stimulus; e.g., a kitchen scene shown prior to the presentation of a target made it more likely that the target was seen as a loaf of bread rather than a (similarly shaped) rural mailbox. Estes (1975) used a similar technique to insure the perceptibility of the "contextual" letters in his wordsuperiority study; that is, he presented the contextual letters prior to the test letter. The technique employed in the present study seems to be a more natural way of controlling the relative perceptibility of "contextual" and "target" components. Although the large- and small-letter components of the stimuli were presented simultaneously and for the same duration, only the small letters were difficult to distinguish. This seems analogous to the relative perceptibility of various levels of form in a natural visual image, and the integration of redundant information in such forms, a natural component of the perceptual process. In any case, while Palmer's approach differs in a number of respects from this author's, he does develop a very interesting analysis of his contextual effects based on Morton's (1970) logogen model.

\section{"Level of Form" and "Order of Processing"}

Many of the early computer pattern recognition routines employed a "feature extraction" stage followed by a synthesis of higher order forms (e.g., Guzman, 1969). These "features" were such things as "lines" and "vertices," etc., from which higher order forms, such as cubes or surfaces, could be identified. Similar ("bottom-up") processes have been proposed in psychological theories of tachistoscopic letter recognition (e.g., Rumelhart, 1970; Shiffrin \& Geisler, 1973). Letters were "constructed" or identified from previously "extracted" "features" (e.g., line segments or vertices, etc.). While such processes are quite consistent with a syntactical view of image structure, the spatial frequency view of structure suggests other possibilities. For example, the "gross forms" represented in the low spatial frequency components of an image may be accessible ("coded," "extracted") at the same time as the highfrequency components. In fact, there is some suggestion that low-frequency components may be perceived earlier than high-frequency ones (Navon, 1975; Tolhurst, 1975). Thus the temptation to think of "features" as "low-order forms" (such as line segments, vertices, etc.) may be misleading. If, as has been suggested by some investigators (e.g., Campbell \& Robson, 1968), the visual system performs a spatial Fourier analysis on images, the "features" of our perceptual process may correspond to activation of certain frequency-specific systems. Thus both levels of form may be processed in parallel, rather than in the sequential manner suggested by the syntactic analysis of images. There is some precedent for assuming an early lowfrequency analysis of images. Many computer processing schemes involve an early "formatting" or "segmentation" of the image into units to be processed in detail. Psychologists have also considered this issue (e.g., Kinchla, 1974; Reed, 1973), although it is often obscured in studies where the stimulus arrays have fixed formats on every test trial, such as a 4 by 4 matrix of letters. Repeated practice with fixed formats might "automatize" the early "segmentation" or "chunking" process to an extent that it may be essentially ignored. For example, Rumelhart (1970) simply asserts that each "letter", in a matrix is assigned a "channel" during a "feature extraction" phase, without considering how the observer decides how many "channels" are appro- 
priate. The type of stimuli employed in the present experiment (Figure 1) clearly indicate the complexity of this problem; e.g., does the observer simultaneously "segment" or "chunk" the array into 10 small letters ("channels") and 2 large ones? This author has previously suggested a possible conflict between organizing ("segmenting," "chunking") the large and small letters in arrays of this sort (Kinchla, 1974). Some very interesting work along this line has been reported by Banks and Prinzmetal (1972). They have shown that "higher order" forms produced by arrangements of smaller letters influence the interactions between these letters beyond that attributable to simple proximity (e.g., Wolford, 1975). Again, whether the "organization" of higher order forms precedes, follows, or is simultaneous with the processing of lower order forms seems entirely open at this point. It is clear that much more research is required on how levels of organization or "form" interact in visual processing.

\section{REFERENCE NOTE}

1. Kinchla, R. A. Temporal uncertainty in multiple-observation detection tasks. Technical Report No. 2, Department of Psychology, McMaster University, Hamilton, Ontario, Canada, 1968.

\section{REFERENCES}

Banks, W. P., \& Prinzmetal, W. Configurational effects in visual information processing. Perception \& Psychophysics, 1972, 12, 278-286.

Brondbent, D. E. The hidden preattentive processes. American Psychologist, 1977, 32, 109-118.

CAmprell, F. W., \& Robson, J. G. Application of Fourier analysis to the visibility of gratings. Journal of Physiology (London), 1968, 197, 551-566.

ERIKSEN, C. W., \& SPENCER, T. Rate of information processing in visual perception: Some results and methodological considerations. Joumal of Experimental Psychology Monograph, 1969, 79, No. 2 , Pt. 2.

Estes, $\mathbf{W}$. K. The locus of inferential and perceptual processes in letter identification. Journal of Experimental Psychology: General, 1975, 104, 122-145.

Estes, W. K., \& TAYloR, H. A. Visual detection in relation to display size and redundancy of critical elements. Perception \& Psychophysics, 1966, 1, 9-16.

Fu. K. S. Syntactic methods in pattern recognition. New York: Academic Press, 1974.

GARDNER, G. T. Evidence for independent parallel channels in tachistoscopic perception. Cognitive Psychology, 1973, 4, 130-155.

Guzman, A. Decomposition of a visual scene into threedimensional bodies. In A. Grasselli (Ed.), Automatic interpretation and classification of images. New York: Academic Press. 1969.

Johnston, J. C., \& McClelland, J. L. Perception of letters in words: Seek not and ye shall find. Science, 1974, 184, 1192-1194.

KINCHLA, R. A. Temporal and channel uncertainty in detection: A multiple-observation analysis. Perception \& Psychophysics, $1969,5,129-136$.

KINCHLA, R. A. Detecting target elements in multielement arrays: A confusability model. Perception \& Psychophysics, 1974, 15, 149-158.

Kinchl A, R. A., \& Collyer, C. Detecting target letters in briefly presented arrays: A confidence rating analysis in terms of : weighted additive effects model. Perception \& Psychophysics, 1974, 16, 117-122.

Morton, J. A functional model for memory. In D. A. Norman (Ed.), Models of human memory. New York: Academic Press, 1970.

Navon, D. Global precedence in visual recognition. Unpublished doctoral dissertation, University of San Diego, 1975.

PALMER, S. E. The effects of contextual scenes on the identification of objects. Memory \& Cognition, 1975, 3, 519-526.

ReEd, S. K. Psychological processes in pattern recognition. New York: Academic Press, 1973.

Rumelhart, D. E. A multi-component theory of the perception of briefly exposed visual displays. Joumal of Mathematical Psychology, 1970, 7, 191-218.

SChNeIder, W., \& Shiffrin, R. M. Controlled and automatic human information processing. Psychological Review, 1977, in press.

ShIFFRIN, R. M., \& GARDNER, G. T. Visual processing capacity and attentional control. Journal of Experimental Psychology, 1972, 93, 72-82.

SHIfFrin, R. M., \& GeIsLeR, W. S. Visual recognition in a theory of information processing. In $R$. L. Solso (Ed.), Contemporary issues in cognitive psychology: The Loyola symposium. Washington, D.C: Winston, 1973.

Shiffrin, R. M., \& SChNELDER, W. Controlled and automatic human information processing: II. Psychological Review, 1977, in press.

SPERLiNg, G. A. A model for visual memory tasks. Human Factors, $1963,5,19-31$.

Tolmurst, D. J. Reaction times in the detection of gratings by human observers: A probabilistic mechanism. Vision Research, 1975, 15, 19-31.

WHEELER, D. D. Processes in word recognition. Cognitive Psychology, 1970, 1, 59-85.

WOLFORD, G. Perturbation model for letter identification. Psychological Review, 1975, 82, 184-199:

\section{NOTES}

1. In addition to the Kinchla (1974) paper, a description of these stimuli and some of the work reported here was presented by this author at a Workshop on Pattern Recognition sponsored by the National Science Foundation at Stanford University in August of 1973. Navon (1975) employed similar stimuli in a series of excellent experiments bearing on the order in which components of an image are processed.

2. For example, suppose each letter, $e_{i}$, in an n-letter array $\left(e_{1}, e_{2}, \ldots e_{n}\right)$ evoked a "subjective impression," $X_{i}$, where each $X_{i}$ was a Gaussian random variable with a fixed variance, $a$, and an expected value equal to 1 if $e_{i}$ was a target letter or 0 if it was a nontarget letter. If observers based their response on the sum of the evoked impressions $\left(\Sigma X_{i}\right)$, each additional nontarget letter would add nothing to the expected value of the sum, but would increment the total variance of that sum by the amount $\alpha$. Thus a $\mathrm{d}^{\prime}$ discriminability measure for detecting one target among $n$ letters would equal $1 / \alpha^{1 / 2}$ for single-letter arrays or $1 /(\mathrm{n} a)^{1 / 2}$ for $n$ letter arrays; i.e., each nontarget letter diminishes $d^{\prime}$ even though the processing of each letter is characterized by the same letter-conditional distributions of $\mathrm{X}$.

3. It can be shown that the estimates of $\alpha_{\mathrm{L}}$ and $\alpha_{\mathrm{T}}$ derived from the "L-only" and "T-only". data provide a better $\left(\chi^{2}<.01\right)$ account of each observer's data from the "other" condition than would values of $\alpha_{\mathrm{L}}$ and $\alpha_{\mathrm{T}}$ only $10 \%$ larger. Thus, if there is an attentional problem in the either condition (where the observer has to share "processing capacity" between both large letters), it must involve less than a $10 \%$ increase in $\alpha_{\mathrm{L}}$ and $\alpha_{\mathrm{T}}$.

(Received for publication January 17, 1977; accepted March 21, 1977.) 